\title{
Effects of $\mathbf{T}$ cell immunoglobulin and mucin domain-containing molecule-3 signaling molecule on human monocyte-derived dendritic cells with hepatitis B virus surface antigen stimulation in vitro
}

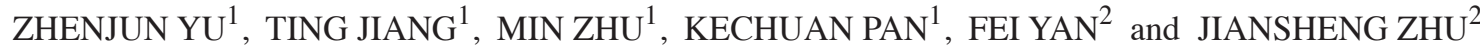 \\ ${ }^{1}$ Medical Research Center of Taizhou Hospital, Wenzhou Medical University; ${ }^{2}$ Department of Infectious Diseases, \\ Affiliated Taizhou Hospital of Wenzhou Medical University, Linhai, Zhejiang 317000, P.R. China
}

Received February 5, 2015; Accepted December 11, 2015

DOI: $10.3892 / \mathrm{mmr} .2016 .4815$

\begin{abstract}
The aim of the present study was to investigate the in vitro effects of hepatitis B virus surface antigen (HBsAg) on the immune function of human monocyte-derived dendritic cells (MD-DCs), and the moderating role of $\mathrm{T}$ cell immunoglobulin and mucin domain-containing molecule-3 (Tim-3) signaling molecule. The monocytes, obtained from healthy adult peripheral blood, were incubated with recombinant human granulocyte-macrophage colony-stimulating factor and interleukin (IL)-4 to induce DCs. DC-associated cell markers were detected using flow cytometry. MD-DCs were treated with HBsAg $(5 \mu \mathrm{g} / \mathrm{ml})$ in vitro for $48 \mathrm{~h}$ and subsequently, cell markers, lymphocyte stimulatory capacity, signaling protein and downstream cytokines were assessed. In addition, a Tim-3 monoclonal antibody was used to inhibit the Tim-3 signaling pathway, and subsequently the immune responses of MD-DCs to HBsAg stimulation were determined using the aforementioned method. The cell phenotype expressions of MD-DCs were all significantly increased with cluster of differentiation (CD)11c at 70.09 $\pm 0.57 \%$, human leukocyte antigen-DR at $79.83 \pm 2.12 \%, \mathrm{CD} 80$ at $48.33 \pm 7.34 \%$ and CD86 at $44.21 \pm 5.35 \%$. The treatment of MD-DCs with
\end{abstract}

Correspondence to: Mr. Jiansheng Zhu, Department of Infectious Diseases, Affiliated Taizhou Hospital of Wenzhou Medical University, 150 Ximen Road, Linhai, Zhejiang 317000, P.R. China E-mail: zhujs126@126.com

Abbreviations: BSA, bovine serum albumin; $\mathrm{CHB}$, chronic hepatitis B; DCs, dendritic cells; MD-DCs, monocyte derived DCs; $\mathrm{HBV}$, hepatitis B virus; HBsAg, HBV surface antigen; IFN, interferon; $\mathrm{NF}-\kappa \mathrm{B}$, nuclear factor $\kappa \mathrm{B}$; NK, nature killer cell; PBMCs, peripheral blood mononuclear cells; Th1, T helper 1 cells; TH17, T helper 17 cells; SI, stimulation index; Tim-3, T cell immunoglobulin and mucin domain-containing molecules-3.

Key words: dendritic cells, hepatitis B virus surface antigen, immune response, nuclear factor- $\kappa \mathrm{B}, \mathrm{T}$ cell immunoglobulin, mucin domain-containing molecules-3
HBsAg resulted in a CD80 and CD86 enhanced expression, enhanced lymphocyte stimulatory capacity, upregulated expression of Tim-3 and nuclear factor $-\kappa \mathrm{B}(\mathrm{NF}-\kappa \mathrm{B})$, as well as enhanced cytokine secretion of IL-6, IL-10 and interferon (IFN) $-\gamma$. However, a reduced immune response of MD-DCs in response to HBsAg stimulation was observed when the Tim-3 signaling pathway was inhibited prior to stimulation. The expression of $\mathrm{NF}-\kappa \mathrm{B}$ was decreased and the cytokine secretion level of IL-6, IL-10 and IFN- $\gamma$ were downregulated. The treatment with HBsAg in vitro resulted in an enhanced immune response of MD-DCs, which may be positively regulated by the Tim-3 signaling molecule.

\section{Introduction}

Hepatitis B virus (HBV) infection, an infection that can cause chronic liver disease, and which raises the risks of mortality from liver cirrhosis and cancer, is a major public health problem worldwide (1). It is estimated that $\sim 2$ billion individuals worldwide exhibited serological evidence of current or past HBV infection, and $\sim 350,000,000$ individuals are suffering from chronic HBV infection (2). As a non-cytopathic virus, HBV fails to directly destroy the host cells, however, it could influence disease progression and prognosis by activating the innate or adaptive immune response (3). Dendritic cells (DCs), which are powerful antigen-presenting cells in vivo, have been shown to form a critical interface between the innate and adaptive immune response (4), and serve as important components of the regulation of immune responses (5). DC dysfunction in patients with chronic HBV (CHB), which can cause inefficient antigen presenting and inadequate special antiviral immune response, was an important reason for persistent infection of HBV (6). In addition, previous studies have suggested that the significantly lower frequency of myeloid DCs (mDCs) and plasmacytoid DCs (pDCs) in the umbilical cord blood, compared with that in the peripheral blood of healthy adults, can comprise the mechanism of easily-acquired chronicity among newborns following HBV infection (7).

Large quantities of HBV particles and viral proteins, including the hepatitis B virus surface antigen ( $\mathrm{HBsAg}$ ), were identified in the peripheral blood of $\mathrm{HBV}$-infected 
patients. The HBsAg can accumulate up to concentrations of $100 \mu \mathrm{g} / \mathrm{ml}$ (8). In acute HBV infection, the cellular and humoral immune response induced by HBsAg may be important in the control of HBV replication and life cycle (9). In addition, it has previously been suggested that HBsAg can alter the immune response, which may contribute to the establishment of chronic infection; Wang et al (10) demonstrated an association between HBsAg and decreased cytokine production, in particular that of interleukin (IL)-12, which may have been induced via the toll-like receptor 2 ligand in peripheral blood mononuclear cells (PBMCs) from patients with CHB. These findings can lead to the understanding of the mechanism through which HBV evades host immunity. It has also been demonstrated that HBsAg may directly interfere with pDC function through the HBsAg-mediated upregulated expression of the suppressor of cytokine signaling 1 and blood DC antigen-2 ligation $(11,12)$.

$\mathrm{T}$ cell immunoglobulin and mucin domain-containing molecule-3 (Tim-3), a member of the Tim family, was found to be involved in the pathogenesis of numerous diseases, including virus infections, cancer, autoimmune diseases, and also allergic reactions and transplantation rejection (13). Khademi et al (14) first reported the high mRNA expression levels of Tim-3 in human T helper 1 cells (Th1) in vitro, therefore Tim-3 was initially considered as a special membrane protein expressed on the Th1 cells (15); however, it was revealed that Tim-3 was also expressed on other cell types, including cluster of differentiation (CD)8+T, Th17, nature killer (NK), mast, endothelial and regulatory cells, as well as monocytes, macrophages, DCs and neurogliocytes (13). A previous study showed that Tim-3 was also expressed in tumor cells and could serve as an independent prognostic factor for cancer (16).

In the present study, human monocyte-derived DCs (MD-DCs) from normal adult peripheral blood were selected in order to investigate the effects of HBsAg on the immune function of MD-DCs in vitro, and the possible role of the Tim-3 signaling molecule in the regulation of MD-DCs.

\section{Materials and methods}

Generation of MD-DCs. DCs were cultured, as previously described with slight modifications (17). The PBMCs were obtained from healthy adult donors by centrifugation with Ficoll-Paque (Sigma-Aldrich, St. Louis, MO, USA). Written informed consent was obtained from all donors prior to blood donation. The present study was approved by the ethics committee at the Affiliated Taizhou Hospital of Wenzhou Medical University (Linhai, China). The cells were cultured in RPMI-1640 medium in 6-well plates for $2 \mathrm{~h}$ at $37^{\circ} \mathrm{C}$. Non-adherent cells were removed by gently swirling the plate and adherent cells (monocytes) were subsequently cultured in RPMI-1640 medium, supplemented with $20 \%$ heat-inactivated fetal bovine serum (HyClone ${ }^{\mathrm{TM}}$; GE Healthcare Life Sciences, Logan, UT, USA) in 6-well plates with recombinant human granulocyte-macrophage colony-stimulating factor (rhGM-CSF; $750 \mathrm{U} / \mathrm{ml}$; R\&D Systems, Inc., Minneapolis, MN, USA) and rhIL-4 (800 U/ml; R\&D Systems, Inc.). Fresh medium containing GM-CSF and IL-4 was added every 3 days. Phosphate-buffered saline (PBS) was used to replace GM-CSF and IL-4 in the control group.
Flow cytometry. MD-DCs were collected in test tubes, incubated in PBS and stained with fluorescein isothiocyanate (FITC)-phycoerythrin (PE)-allophycocyanin (APC)- or peridinin-chlorophyll-protein (PerCP)-labeled monoclonal antibodies (anti-human CD80, CD86, CD11c and human leukocyte antigen (HLA)-DR, or the relevant isotype control; eBioscience Inc., San Diego, CA, USA), according to the manufacturer's protocol. Following incubation at room temperature in the dark for $15 \mathrm{~min}$, the cells were washed twice with PBS buffer and analyzed using a FACSort cell analyzer (FACSCalibur; Becton-Dickinson, San Jose, CA, USA). The flow cytometry data was analyzed using the FlowJo software, version 7.6.5 (FLOWJO, LLC, Ashland, Oregon, USA).

Treatment of MD-DCs with HBsAg. HBsAg $(5 \mu \mathrm{g} / \mathrm{ml}$; ProSpec-Tany TechnoGene Ltd., Ness Ziona, Israel) was added to the medium of MD-DCs on day 7 following culture, while the control group was treated with $0.2 \%$ bovine serum albumin (BSA). The cells were treated for $48 \mathrm{~h}$.

Inhibition of the Tim-3 signaling pathway. Initially, the Ultra-Leaf purified anti-human Tim-3 (10 $\mu \mathrm{g} / \mathrm{ml}$; BioLegend, San Diego, CA, USA) was added to the medium of MD-DCs on day 7 following culture to inhibit the Tim-3 signaling pathway, while the control group was treated with BSA $(0.2 \%)$. After $1 \mathrm{~h}, \mathrm{HBsAg}(5 \mu \mathrm{g} / \mathrm{ml})$ was added to the medium for $48 \mathrm{~h}$.

Mixed leukocyte reaction. MD-DCs treated with mitomycin C $(25 \mu \mathrm{g} / \mathrm{ml})$ for $30 \mathrm{~min}$, were used as the stimulating cells. Allogenic lymphocytes, which were obtained from other healthy adult donors, as previously described, were distributed at in 96-well plates a density of $1 \times 10^{5}$ cells/well and were incubated for $96 \mathrm{~h}$ in certain ratio of stimulating cells (DC/lymphocytes $=1 / 5$ or $1 / 10)$. MTS-phenazine methosulfate (20 $\mu \mathrm{l} /$ well; Promega Corp., Madison, USA) was added and incubated for $4 \mathrm{~h}$ at $37^{\circ} \mathrm{C}$, after which the absorbance (A) at $450 \mathrm{~nm}$ was determined using a Microplate Reader (CLARIOstar ${ }^{\circledR}$; BMG LABTECH GmbH, Ortenberg, Germany). The stimulation index (SI) was calculated as follows: $\mathrm{SI}=\left(\mathrm{A}_{\text {experimental }}-\mathrm{A}_{\text {background }}\right) /\left(\mathrm{A}_{\text {control }}-\mathrm{A}_{\text {background }}\right)(18)$.

Western blotting. The proteins from the MD-DCs were collected through progressions of pyrolysis and extraction, and the total protein concentration was determined using a bicinchoninic acid protein detection kit (Sigma-Aldrich). The total protein $(40 \mu \mathrm{g})$ was separated using $10 \%$ sodium dodecyl sulfate-polyacrylamide gel electrophoresis (Sigma-Aldrich), and the proteins were transferred onto polyvinylidene difluoride membranes (Sigma-Aldrich). The membranes were first incubated for $2 \mathrm{~h}$ at room temperature with $1 \%$ BSA in PBS, and then overnight at $4^{\circ} \mathrm{C}$ with rabbit anti-human Tim-3 polyclonal antibody (dilution, 1:1,000; cat. no. 3808-100; BioVision, Inc., Milpitas CA, USA), and anti-NF- $\kappa \mathrm{B}$ (dilution, 1:1,000; cat. no. 1559-1; Epitomics, Inc., Burlingame, CA, USA), and anti-GAPDH monoclonal (dilution, 1:1,000; cat. no. 5632-1; Epitomics, Inc.) antibodies. For signal detection, horseradish peroxidase-conjugated mouse anti-rabbit immunoglobulin $\mathrm{G}$ (dilution, 1:10,000; cat. no. 5618-1; Epitomics, Inc.) and the enhanced chemiluminescence detection system (Cell Signaling 


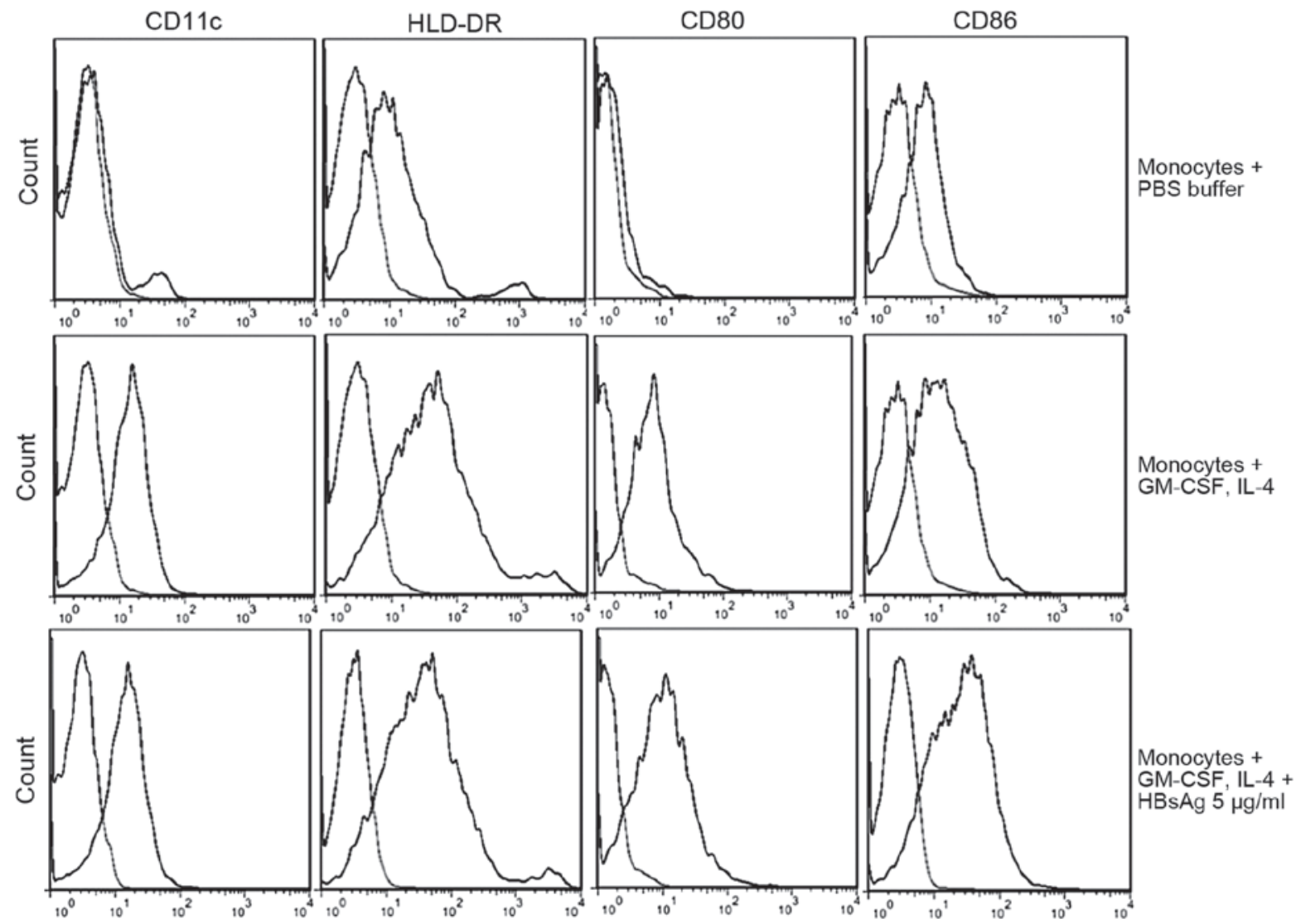

Figure 1. Phenotypic analysis of human monocyte-derived dendritic cells. The phenotypic expression values of monocytes cultured with PBS buffer were as follows: CD11c $(9.41 \pm 6.38 \%)$, HLA-DR $(26.13 \pm 6.61 \%)$, CD80 (3.46 $\pm 2.00 \%)$ and CD86 $(6.70 \pm 3.21 \%)$. The expression of monocytes cultured with GM-CSF and IL-4 was significantly increased (P<0.01): CD11c (70.09 $\pm 0.57 \%)$, HLA-DR $(79.83 \pm 2.12 \%)$, CD80 (48.33 $\pm 7.34 \%)$ and CD86 (44.21 $\pm 5.35 \%)$. In addition, HBsAg treatment with GM-CSF and IL-4, increased the expression of CD80 $(\mathrm{t}=3.75 ; \mathrm{P}<0.05)$ and CD86 $(\mathrm{t}=7.61 ; \mathrm{P}<0.01)$. The cell phenotypic expression values

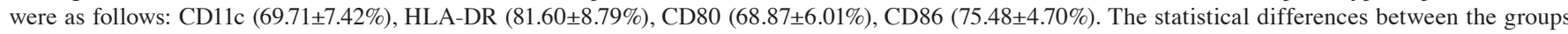
were determined using Student's t-test. The graphs were generated using FlowJo software, version 7.6.5. The light regions on the graphs represent the control samples. GM-CSF, granulocyte-macrophage colony-stimulating factor, HLA, human leukocyte antigen; CD, cluster of differentiation; HBsAg, hepatitis B virus surface antigen; IL, interleukin; PBS, phosphate-buffered saline.

Technology, Inc., Danvers, MA, USA) were used. According to the gray value ratio of target protein against GAPDH, the relative expression of the target protein was calculated using the Quantity One software, version 4.6.2 (Bio-Rad Laboratories, Inc., Hercules, CA, USA), as demonstrated in a previous study (19).

Enzyme-linked immunosorbent assay (ELISA). The concentrations of inflammatory cytokines in the supernatant were determined using the following ELISA kits: Human IL-6 Quantikine ELISA kit, Human IL-10 Quantikine ELISA kit and Human IFN- $\gamma$ Quantikine ELISA kit (R\&D Systems, Inc.). The experiments were performed according to the manufacturer's protocol.

Statistical analysis. Continuous variables are expressed as the mean \pm standard deviation. Student's t-test was used to determine the statistical differences between two groups, one-way analysis of variance for multiple groups and the least significant difference t-test was used for multiple comparisons. Statistical analyses were conducted using the GraphPad Prism software, version 5.01 (GraphPad Software, Inc., La Jolla, CA, USA). $\mathrm{P}<0.05$ was considered to indicate a statistically significant difference.

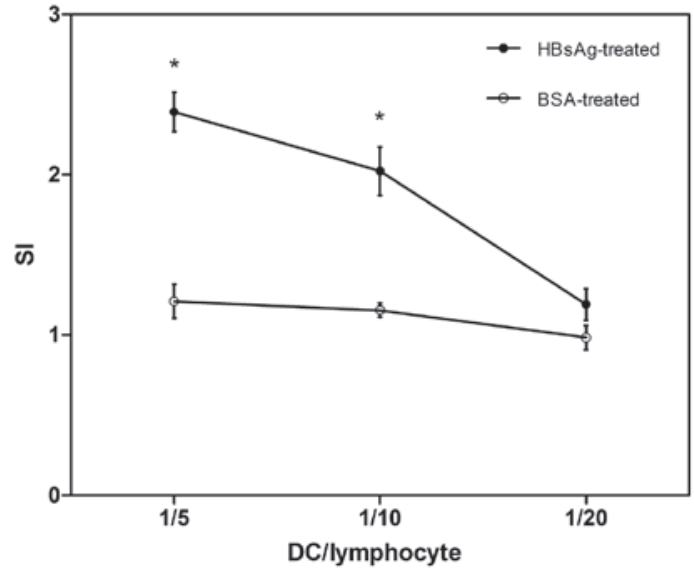

Figure 2. Lymphocyte stimulatory capacity of MD-DCs. A higher SI represented a higher lymphocyte stimulatory capacity. At a $1 / 5$ ratio of DC/lymphocytes, the SI of BSA-treated MD-DCs was $1.21 \pm 0.10$, while that of HBsAg-treated MD-DCs was 2.39 \pm 0.12 . In vitro HBsAg stimulation enhanced the lymphocyte stimulatory capacity of MD-DCs $(t=14.71$; $\left.{ }^{*} \mathrm{P}<0.01\right)$. Similarly, the capacity of MD-DCs was enhanced at the $1 / 10$ ratio of DC/lymphocytes $\left(\mathrm{t}=11.12 ;{ }^{*} \mathrm{P}<0.01\right)$. The SI difference revealed no statistically significant difference when the ratio of DC/lymphocytes was $1 / 20$. Statistical differences between the groups were determined using Student's t-test. Data are presented as the mean \pm standard deviation. SI, stimulation index; MD-DCs, human monocyte-derived dendritic cells; HBsAg, hepatitis B virus surface antigen; BSA, bovine serum albumin 
A
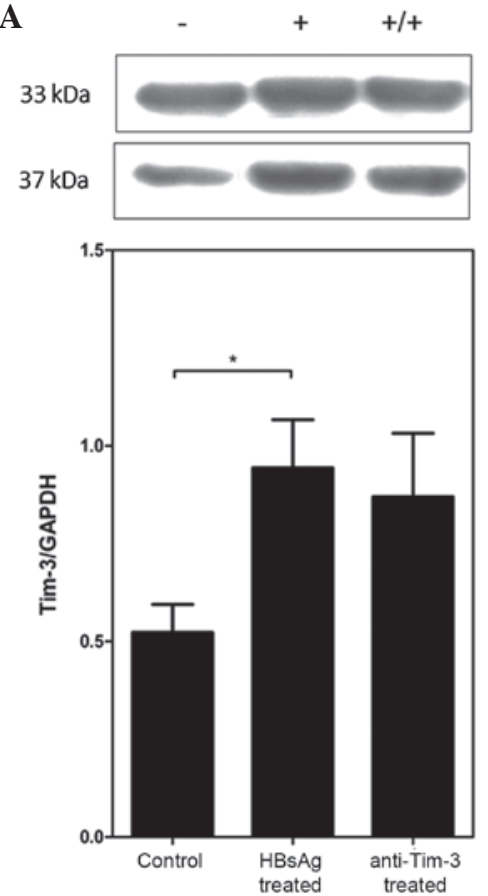

GAPDH

Tim-3
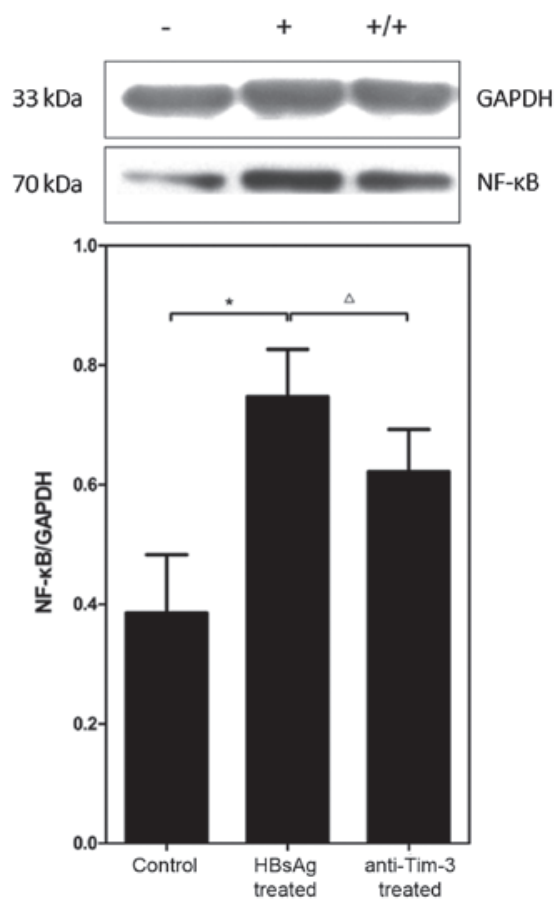

Figure 3. Expression of signaling proteins. The expression of (A) Tim-3 and (B) NF- $\mathrm{kB}$ was determined in the three groups. Following HBsAg stimulation in vitro, the expression of the signaling proteins Tim-3 and NF- $\mathrm{KB}$ was significantly increased in MD-DCs, while a decreased expression of NF- $\mathrm{KB}$ was observed when the Tim-3 signaling pathway was inhibited with anti-Tim-3 prior to HBsAg stimulation. The expression of Tim-3 in the MD-DCs revealed no significant decrease when treated with anti-Tim- 3 , since it was the antagonist of Tim-3 signaling molecule. $\left({ }^{\Delta} \mathrm{P}<0.05\right.$; $\left.{ }^{*} \mathrm{P}<0.01\right)$. Statistical differences between the groups were determined using one-way analysis of variance and the least significant difference t-test. Data are presented as the mean \pm standard deviation.

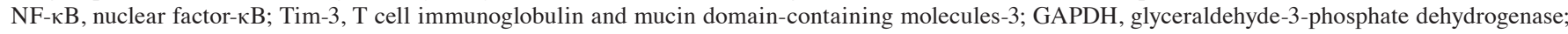
HBsAg, hepatitis B virus surface antigen; MD-DCs, human monocyte-derived dendritic cells; BSA, bovine serum albumin.

\section{Results}

Identification of MD-DCs. Following 7 days of incubation with RPMI-1640 medium containing rhGM-CSF and rhIL-4, the majority of the cells aggregated to form suspended colonies, which were subsequently observed using an optical microscope. Electron microscopy revealed that the morphology of the MD-DCs was irregular, with burr cells observed on the surface. These findings were consistent with the typical DC morphology (20). The expression of DC-associated cell phenotypes was revealed to be significantly increased with CD11c $70.09 \pm 0.57 \%$, HLA-DR $79.83 \pm 2.12 \%$, CD80 $48.33 \pm 7.34 \%$ and CD86 $44.21 \pm 5.35 \%$ ( $\mathrm{P}<0.01$; Fig. 1).

Effects of HBsAg on MD-DCs. Compared with the control group, HBsAg treatment resulted in an enhanced expression of co-stimulatory molecules $(\mathrm{P}<0.05)$ with CD80 $(68.87 \pm 6.01 \%$ ) and $\operatorname{CD} 86(75.48 \pm 4.70 \%)$. The changes in the cell phenotype expression of CD11c and HLA-DR revealed no statistically significant difference ( $\mathrm{P}>0.05$; Fig. 2). A higher stimulation index (SI) represented a more marked lymphocyte stimulatory capacity. The SI of HBsAg-treated MD-DCs was higher compared with the control BSA treated group, with a $1 / 5$ or $1 / 10$ ratio of DC/lymphocytes $(\mathrm{P}<0.01$; Fig. 2). Treatment of the MD-DCs with HBsAg resulted in the upregulation of NF- $\mathrm{KB}(\mathrm{t}=7.55, \mathrm{P}<0.01$; Fig. $3 \mathrm{~B})$, as well as an enhancement in the cytokine secretion levels of IL-6 $(\mathrm{t}=6.05$, $\mathrm{P}<0.01), \mathrm{IL}-10(\mathrm{t}=7.02, \mathrm{P}<0.01)$ and interferon (IFN)- $\gamma(\mathrm{t}=11.69$, $\mathrm{P}<0.01$; Fig. 4).

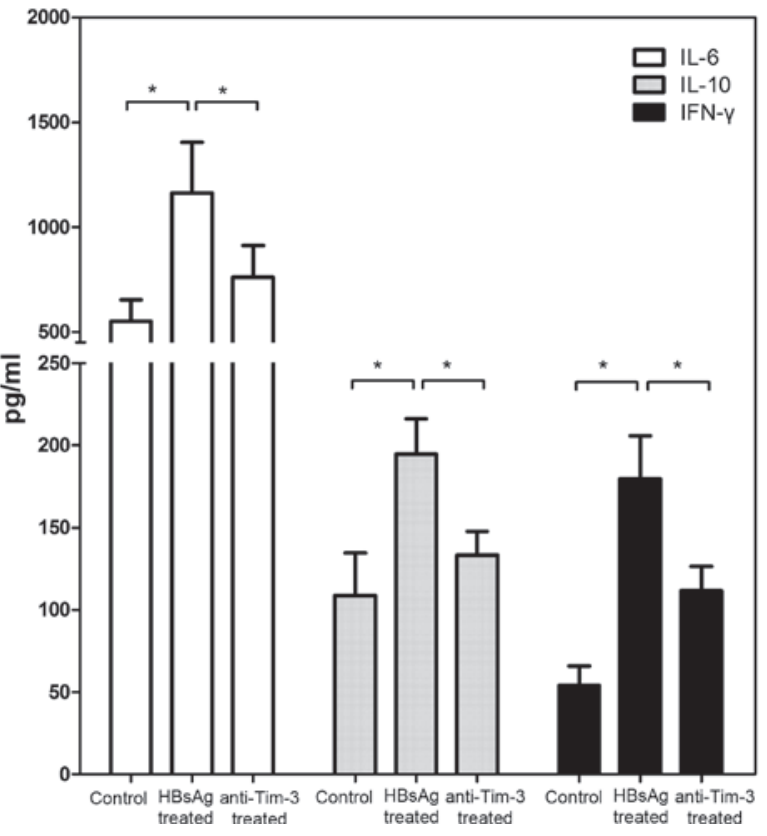

Figure 4. Cytokine secretion levels. Following HBsAg stimulation, in vitro, levels of the downstream cytokines, IL-6, IL-10 and IFN- $\gamma$, were significantly increased, which indicated an enhanced immune response of MD-DCs to HBsAg stimulation. This immune response, was reduced following inhibition of the Tim-3 signaling pathway, using anti-Tim-3 prior to HBsAg stimulation. The secretion of IL-6, IL-10 and IFN- $\gamma$ cytokines was significantly downregulated. ( $\mathrm{P}<0.01)$. Statistical differences between the groups were determined using one-way analysis of variance and the least significant difference t-test. Data are presented as the mean \pm standard deviation. HBsAg, hepatitis B virus surface antigen; IL, interleukin; IFN- $\gamma$, interferon- $\gamma$; MD-DCs, human monocyte-derived dendritic cells; Tim-3, T cell immunoglobulin and mucin domain-containing molecules-3; BSA, bovine serum albumin. 
Role of Tim-3 in the regulation of MD-DCs. HBsAg stimulation in vitro increased the expression of Tim-3 $(\mathrm{t}=5.83, \mathrm{P}<0.01)$ in MD-DCs (Fig. 3A) and enhanced the immune function of MD-DCs; however, reduced the immune response of MD-DCs to HBsAg stimulation was observed when the Tim-3 signaling pathway was inhibited prior to treatment. The NF- $\mathrm{BB}$ expression was revealed to be significantly decreased $(\mathrm{t}=2.61, \mathrm{P}<0.05)$ and the cytokine secretion level of IL- $6(\mathrm{t}=3.99, \mathrm{P}<0.01)$, IL-10 $(\mathrm{t}=5.03, \mathrm{P}<0.01)$ and IFN- $\gamma(\mathrm{t}=6.30, \mathrm{P}<0.01)$ were significantly downregulated (Fig. 4).

\section{Discussion}

The present study demonstrated that the HBsAg enhanced the immune response of MD-DCs in vitro. Previous studies have revealed large quantities of $\mathrm{HBV}$ particles and viral proteins in the peripheral blood of $\mathrm{HBV}$-infected patients, which enabled multiple interactions among the virus, its viral proteins and the immunocytes (21). HBsAg enhanced the innate or adaptive immune response found in the mice bone marrow-derived DCs treated with HBsAg in vitro, as well as affect the differentiation of $\mathrm{T}$ helper cells (22). Different forms of $\mathrm{HBsAg}$ elicited various types of B and T cell immunity (23), and both HBV DNA vaccine and HBsAg induced the humoral immunity (24). In the present study, MD-DCs were treated with HBsAg in vitro, which resulted in the enhanced expression of co-stimulatory molecules and the lymphocyte stimulatory capacity of MD-DCs, as well as the upregulated secretion of inflammatory cytokines. Consistent with the present study, Jan et al (19) also used the MD-DCs from healthy adult donors and reported that HBsAg increases the expression of CD80, CD83, CD86 and major histocompatibility complex class II of MD-DCs. This treatment also increases the production of interleukin IL-12 and IL-10, as well as enhances T cell-stimulatory capacity. In addition, treated with different concentrations of HBsAg, the immature DCs, which were cultured from PBMCs of patients with CHB, differentiate into mature DCs (25); and DCs stimulated with HBsAg more efficiently presented antigen and induced a specific $\mathrm{T}$ cell immune response (26). However, Op den Brouw et al (21) reported that both HBV particles and purified HBsAg had an immune modulatory capacity and directly contributed to the dysfunction of mDCs in patients with chronic HBV; since the mDCs were directly isolated from PBMCs, the differences between circulating mDCs and MD-DCs in immune function, as well as the exact molecular association between the HBsAg and DCs, remained unclear.

In the present study, in vitro HBsAg stimulation increased the expression of the Tim-3 signal molecule and enhanced the immune function of MD-DCs. Tim-3 was expressed in a variety of immune cells, exerts an important role in regulating acquired or innate immune responses, and was revealed to be closely associated with the pathogenesis of infection, cancer and other diseases (27). The interaction between Tim-3 and its natural ligand, galectin-9, induces intracellular calcium efflux, resulting in Th1 cell aggregation and apoptosis, followed by immune tolerance and inhibition of acquired immune response (15). In the innate immune response, Tim-3 acts as a promoter or inhibitor through its interaction with galectin-9 or other ligands. Anderson et al (28) reported that Tim-3, expressed on antigen presenting cells, cooperates with Toll-like receptors to promote the secretion of proinflammatory cytokines. Kanzaki et al (29) reported that the galectin-9-Tim-3 signaling pathway induces TNF- $\alpha$ production in cultured DCs in a dose-dependent manner; however, Chiba et al (30) revealed that the high expression of Tim-3 on tumor-associated DCs inhibited the immune response mediated by nucleic acid. Therefore, different immune environments may lead to different roles of Tim-3-mediated immune response.

The Tim-3 signal molecule may positively regulate the immune response of MD-DCs. As described in the present study, a reduced immune response of MD-DCs to HBsAg stimulation was observed when the Tim-3 signaling pathway was inhibited in advance; however, this conclusion was not consistent with previously published results. Ju et al (31) found that the inhibition of the Tim-3 signaling pathway with anti-Tim-3 antibodies or Tim-3-Fc fusion proteins resulted in an increased cytotoxicity for NK92 cells and an elevated IFN- $\gamma$ production. The increased expression of Tim-3 on PBMCs, circulating NK and CD8+T cells in patients with CHB led to immune response dysfunction. In addition, partial restoration of immune function was observed when the Tim-3 signaling pathway was inhibited prior to treatment $(31,32)$. The opposite regulatory effects of Tim-3 may be attributed to the relatively different immune environment between MD-DCs with simple HBsAg stimulation and patients with chronic HBV infection; however, the exact mechanism remains to be elucidated.

In conclusion, in vitro $\mathrm{HBsAg}$ treatment resulted in an enhanced immune response of MD-DCs, which may be positively regulated by Tim-3. However, further study is required in order to determine the regulatory role of Tim-3 on the immune function of DCs in the intracorporeal immune environment of patients with $\mathrm{CHB}$ and the exact mechanism of Tim-3-mediated regulation.

\section{Acknowledgments}

The present study received financial support from the Zhejiang Bureau of Public Health (no. 2012KYB237) and the Zhejiang Province Major Science and Technology Programs (no. 2012C13018-3). The authors would also like to thank the staff of the Medical Research Center for their helpful advice and guidance.

\section{References}

1. Trépo C, Chan HL and Lok A: Hepatitis B virus infection. Lancet 384: 2053-2063, 2014

2. Hatzakis A, Van Damme P, Alcorn K, Gore C, Benazzouz M, Berkane S, Buti M, Carballo M, Cortes Martins H, Deuffic-Burban S, et al: The state of hepatitis B and C in the Mediterranean and Balkan countries: Report from a summit conference. J Viral Hepat 20 (Suppl 2): 1-20, 2013.

3. Hösel M, Quasdorff M, Wiegmann K, Webb D, Zedler U, Broxtermann M, Tedjokusumo R, Esser K, Arzberger S, Kirschning CJ, et al: Not interferon, but interleukin-6 controls early gene expression in hepatitis B virus infection. Hepatology 50: 1773-1782, 2009.

4. Mildner A and Jung S: Development and function of dendritic cell subsets. Immunity 40: 642-656, 2014.

5. Hänninen A: New dimensions for dendritic cells. Duodecim 130: 883-891, 2014 (In Finnish) 
6. Wang FS, Xing LH, Liu MX, Zhu CL, Liu HG, Wang HF and Lei ZY: Dysfunction of peripheral blood dendritic cells from patients with chronic hepatitis B virus infection. World J Gastroenterol 7: 537-541, 2001.

7. Hao HX, Zhang YL, Li MH, Zhang LX, Yi W, Hu YH, Yi N, Cheng J, Liu SA and Xie Y: Dendritic cell subsets and function in newborns from mothers of different HBV infection status. Zhong Hua Shi Yan He Lin Chuang Bing Du Xue Za Zhi 27: 112-114, 2013 (In Chinese)

8. Ganem D and Prince AM: Hepatitis B virus infection-natural history and clinical consequences. N Engl J Med 350: 1118-1129, 2004.

9. Chen Z, Cheng Y, Xu Y, Liao J, Zhang X, Hu Y, Zhang Q, Wang J, Zhang Z, Shen F and Yuan Z: Expression profiles and function of Toll-like receptors 2 and 4 in peripheral blood mononuclear cells of chronic hepatitis B patients. Clin Immunol 128: 400-408, 2008.

10. Wang S, Chen Z, Hu C, Qian F, Cheng Y, Wu M, Shi B, Chen J, Hu Y and Yuan Z: Hepatitis B virus surface antigen selectively inhibits TLR2 ligand-induced IL-12 production in monocytes/macrophages by interfering with JNK activation. J Immunol 190: 5142-5151, 2013.

11. Xu Y, Hu Y, Shi B, Zhang X, Wang J, Zhang Z, Shen F, Zhang Q, Sun S and Yuan Z: HBsAg inhibits TLR9-mediated activation and IFN-alpha production in plasmacytoid dendritic cells. Mol Immunol 46: 2640-2646, 2009.

12. Kondo Y,Ninomiya M, Kakazu E, Kimura O and Shimosegawa T: Hepatitis B surface antigen could contribute to the immunopathogenesis of hepatitis B virus infection. ISRN Gastroenterol 2013: 935295, 2013.

13. Zhang XM and Shan NN: The role of T cell immunoglobulin and mucin domain-3 in immune thrombocytopenia. Scand J Immunol 79: 231-236, 2014

14. Khademi M, Illés Z, Gielen AW, Marta M, Takazawa N, Baecher-Allan C,Brundin L,HannerzJ,Martin C, Harris RA, et al: T Cell Ig- and mucin-domain-containing molecule-3 (TIM-3) and TIM-1 molecules are differentially expressed on human Th1 and Th2 cells and in cerebrospinal fluid-derived mononuclear cells in multiple sclerosis. J Immunol 172: 7169-7176, 2004

15. Zhu C, Anderson AC, Schubart A, Xiong H, Imitola J, Khoury SJ, Zheng XX, Strom TB and Kuchroo VK: The Tim-3 ligand galectin-9 negatively regulates $\mathrm{T}$ helper type 1 immunity. Nat Immunol 6: 1245-1252, 2005.

16. Jiang J, Jin MS, Kong F, Cao D, Ma HX, Jia Z, Wang YP, Suo J and Cao X: Decreased galectin-9 and increased Tim-3 expression are related to poor prognosis in gastric cancer. PLoS One 8: e81799, 2013.

17. Chapuis F, Rosenzwajg M, Yagello M, Ekman M, Biberfeld P and Gluckman JC: Differentiation of human dendritic cells from monocytes in vitro. Eur J Immunol 27: 431-441, 1997.

18. van derMolen RG, Sprengers D, Binda RS, de Jong EC, Niesters HG Kusters JG, Kwekkeboom J and Janssen HL: Functional impairment of myeloid and plasmacytoid dendritic cells of patients with chronic hepatitis B. Hepatology 40: 738-746, 2004.

19. Jan RH, Lin YL, Chen CJ, Lin TY, Hsu YC, Chen LK and Chiang BL: Hepatitis B virus surface antigen can activate human monocyte-derived dendritic cells by nuclear factor kappa B and p38 mitogen-activated protein kinase mediated signaling. Microbiol Immunol 56: 719-727, 2012.
20. Romani N, Reider D, Heuer M, Ebner S, Kämpgen E, Eibl B, Niederwieser D and Schuler G: Generation of mature dendritic cells from human blood. An improved method with special regard to clinical applicability. J Immunol Methods 196: 137-151, 1996.

21. Op den Brouw ML, Binda RS, van Roosmalen MH, Protzer U, Janssen HL, van der Molen RG and Woltman AM: Hepatitis B virus surface antigen impairs myeloid dendritic cell function: A possible immune escape mechanism of hepatitis B virus. Immunology 126: 280-289, 2009.

22. Jan RH, Lin YL, Chen LK, Huang MT, Wang LC and Chiang BL: Hepatitis B virus surface antigen can activate dendritic cells and modulate $\mathrm{T}$ helper type immune response. Microbiol Immunol 55: 51-59, 2011.

23. Shen M, Wang S, Ge G, Xing Y, Ma X, Huang Z and Lu S: Profiles of $\mathrm{B}$ and $\mathrm{T}$ cell immune responses elicited by different forms of the hepatitis B virus surface antigen. Vaccine 28: 7288-7296, 2010.

24. Du X, Wang J, Kang Y, Xiao W, Zhao G and Wang B: Suppression of the antigen-specific $\mathrm{T}$ cell immune response by co-immunization with the HBV DNA vaccine and recombinant HBsAg. Wei Sheng Wu Xue Bao 49: 938-942, 2009 (In Chinese).

25. Tong HS, Zhang Y, Yuan K and Fu XW: HBsAg loading on dendritic cells in patients with chronic hepatitis B: Expressions of phenotypic molecules. Hepatobiliary Pancreat Dis Int 5: 56-59, 2006.

26. Kang P, Luo SL and Li SC: Comparative study on dendritic cells stimulated with $\mathrm{HBsAg}$ or $\mathrm{HBcAg}$ in patients with chronic hepatitis B. Zhong Hua Shi Yan He Lin Chuang Bing Du Xue Za Zhi 21: 250-252, 2007 (In Chinese).

27. Han G, Chen G, Shen B and Li Y: Tim-3: An activation marker and activation limiter of innate immune cells. Front Immunol 4: 449, 2013.

28. Anderson AC, Anderson DE, Bregoli L, Hastings WD, Kassam N, Lei C, Chandwaskar R, Karman J, Su EW, Hirashima M, et al: Promotion of tissue inflammation by the immune receptor Tim-3 expressed on innate immune cells. Science 318: 1141-1143, 2007.

29. Kanzaki M, Wada J, Sugiyama K, Nakatsuka A, Teshigawara S, Murakami K, Inoue K, Terami T, Katayama A, Eguchi J, et al: Galectin-9 and T cell immunoglobulin mucin-3 pathway is a therapeutic target for type 1 diabetes. Endocrinology 153: 612-620, 2012.

30. Chiba S, Baghdadi M, Akiba H, Yoshiyama H, Kinoshita I, Dosaka-Akita H,Fujioka Y, Ohba Y, Gorman JV, Colgan JD, et al: Tumor-infiltrating DCs suppress nucleic acid-mediated innate immune responses through interactions between the receptor TIM-3 and the alarmin HMGB1. Nat Immunol 13: 832-842, 2012.

31. Ju Y, Hou N, Meng J, Wang X, Zhang X, Zhao D, Liu Y, Zhu F, Zhang L, Sun W, et al: T cell immunoglobulin- and mucin-domain-containing molecule-3 (Tim-3) mediates natural killer cell suppression in chronic hepatitis B. J Hepatol 52: 322-329, 2010.

32. Wu W, Shi Y, Li S, Zhang Y, Liu Y, Wu Y and Chen Z: Blockade of Tim-3 signaling restores the virus-specific $\mathrm{CD}^{+} \mathrm{T}$-cell response in patients with chronic hepatitis B. Eur J Immunol 42: 1180-1191, 2012. 